\title{
Magnetothermoelectric Response at a Superfluid-Mott-Insulator Transition
}

\author{
M. J. Bhaseen, ${ }^{1}$ A. G. Green, ${ }^{2}$ and S. L. Sondhi ${ }^{3}$ \\ ${ }^{1}$ Rudolf Peierls Centre for Theoretical Physics, 1 Keble Road, Oxford, OXI 3NP, United Kingdom \\ ${ }^{2}$ School of Physics and Astronomy, University of St. Andrews, North Haugh, St. Andrews, Fife, KY16 9XP, United Kingdom \\ ${ }^{3}$ Department of Physics, Princeton University, Princeton, New Jersey 08544, USA
}

(Received 26 October 2006; published 18 April 2007; publisher error corrected 18 April 2007)

\begin{abstract}
We investigate the finite temperature magnetothermoelectric response in the vicinity of a superfluid-Mott-insulator quantum phase transition. We focus on the particle-hole symmetric transitions of the Bose-Hubbard model, and combine Lorentz invariance arguments with quantum Boltzmann calculations. By means of an epsilon expansion, we find that a nonvanishing thermoelectric tensor and a finite thermal transport coefficient are supported in this quantum critical regime. We comment on the singular Nernst effect in this problem.
\end{abstract}

DOI: 10.1103/PhysRevLett.98.166801

Since the discovery of high temperature superconductivity, quantum phase transitions between Mott insulators and superfluids (SFs) or superconductors have been the focus of intense scrutiny. More recently, remarkable advances in cold atomic gases have allowed the observation of such phase transitions in systems of bosonic atoms [1]. These transitions separate two of the most fascinating highly correlated states of matter: the superfluid, which reveals the dramatic consequences of quantum coherence on the macroscopic scale, and the Mott insulator, which affirms the impact of strong interactions. In particular, a continuous quantum phase transition between the two clearly requires a combined treatment of strong quantum fluctuations and interactions. Understanding this interplay is central not only to the particular systems at hand, but to a wide variety of condensed matter problems [2]. Unfortunately, such strongly correlated regimes are notoriously difficult to analyze theoretically, and a complete characterization of these transitions is currently lacking.

Our general objective in this work is to advance the theory of continuous superfluid-Mott-insulator (SF-MI) transitions in bosonic systems. In particular, we wish to focus on their quantum critical regimes, and to examine their response to the full array of electric and magnetic fields, and temperature gradients. We are motivated by measurements of such magnetothermoelectric response by Ong and co-workers [3,4]. These have yielded crucial insights into the importance of superconducting fluctuations in the cuprates [5], although a direct correspondence with our present bosonic system is clearly lacking. Our results apply most simply to charged bosons, although there are plausible suggestions to generate pseudomagnetic fields in neutral atomic gases; see, e.g., [6].

Specifically, we focus on the most straightforward example of a SF-MI quantum phase transition and its quantum critical regime - the particle-hole symmetric version occurring at the tips of the Mott lobes in the ubiquitous Bose-Hubbard model [2]. This model arises in numerous contexts including ${ }^{4} \mathrm{He}$ layers, thin film superconductors, and Josephson junction arrays. This system has also been
PACS numbers: 72.20.Pa, 74.25.Fy

realized in groundbreaking experiments on atomic gases [1], where it is possible to tune through the SF-MI transition using lasers. This approach confirms the phase diagram [7] and offers a unique handle on these strongly correlated systems.

In view of this broad spectrum of applications, the BoseHubbard model has received considerable theoretical attention; see, for example, [7-12], and references therein. In this present work we obtain a description of the quantum critical region subject to the full complement of electrical, magnetic, and thermal probes. This opens up new regimes of behavior and brings many more probes within reach. This Letter is structured as follows: We begin with a short introduction to the Bose-Hubbard model and its quantum phase transitions. We recall the quantum Boltzmann equation (QBE) and the epsilon expansion used to access the finite temperature critical dynamics [10]. We briefly discuss the response to a single external field, and then move on to the situation in crossed electric and magnetic fields. We combine Lorentz invariance arguments with Boltzmann calculations to provide the fundamental response coefficients. We then examine the complementary situation in a crossed thermal gradient and a magnetic field. We conclude with implications for possible Nernst measurements, a summary, and directions for further research.

Model. - The Bose-Hubbard model describes bosons hopping on a lattice with amplitude $t$, and interacting via the short range repulsive interaction $U$ :

$$
H=-t \sum_{\langle i j\rangle}\left(b_{i}^{\dagger} b_{j}+b_{j}^{\dagger} b_{i}\right)-\mu \sum_{i} n_{i}+\frac{U}{2} \sum_{i} n_{i}\left(n_{i}-1\right) .
$$

The Bose creation and annihilation operators satisfy the usual commutation relations, $\left[b_{i}, b_{j}^{\dagger}\right]=\delta_{i j}, n_{i}=b_{i}^{\dagger} b_{i}$ is the number of bosons at site $i$, and $\mu$ is the chemical potential. In the context of a Josephson array or superconductor, the bosons represent Cooper pairs of charge $Q=$ $2 e$, tunneling between superconducting regions. In general, one may also include the effects of disorder and long range interactions into such a model, but here we shall concen- 
trate on the simplest case (1). The phase diagram of the Bose-Hubbard model exhibits both superfluid and Mott insulating regions [7], the latter occurring for strong enough repulsive interactions. As a function of the chemical potential $\mu$, this model exhibits a series of Mott insulating "lobes" where the density of bosons is pinned to successive integers. At the tips of these lobes, the energy cost to producing particle-hole excitations vanishes and the model is particle-hole symmetric. In the vicinity of these points, the SF-MI transition is described by the action of a complex scalar field $\Phi[7]$,

$$
S=\int d^{D} x\left|\partial_{\mu} \Phi\right|^{2}-m^{2}|\Phi|^{2}-\frac{u_{0}}{3 !}|\Phi|^{4},
$$

where $D=d+1$, and $d$ is the number of spatial dimensions. This is nothing but a quantum Landau-Ginzburg theory for the superconducting order parameter $\Phi$. Away from these particle-hole symmetric points, the action picks up an additional term linear in the time derivative. Correspondingly, the dynamical exponent changes from $z=1$ to $z=2$ [2]. Here, we will focus on the case with $z=1$.

Quantum Boltzmann equation. - In the absence of interactions, the model (2) is the celebrated Klein-Gordon theory. The two modes of opposite charge correspond to density fluctuations, or particle-hole excitations, of the Mott insulator. Performing this mode expansion on the full model (2) allows one to develop a Boltzmann approach to transport which is particularly simple at nonzero temperatures and small applied fields [10]. The resulting quantum Boltzmann equation is a nonlinear integrodifferential equation for the distribution functions $f_{ \pm}(\mathbf{k}, t)$ of particle and hole excitations

$$
\frac{\partial f_{ \pm}}{\partial t} \pm Q\left(\mathbf{E}+\mathbf{v}_{\mathbf{k}} \times \mathbf{B}\right) \cdot \frac{\partial f_{ \pm}}{\partial \mathbf{k}}=I_{ \pm}\left[f_{+}, f_{-}\right],
$$

where $\mathbf{v}_{\mathbf{k}} \equiv \partial \varepsilon_{\mathbf{k}} / \partial \mathbf{k}$ and $\varepsilon_{\mathbf{k}} \equiv \hbar \omega_{k}=\sqrt{\mathbf{k}^{2}+m^{2}}$. For simplicity we consider a spatially homogeneous system in uniform external fields. The collision term represents scattering between these excitations, and incorporates the nonlinear interaction of the critical Landau-Ginzburg theory (2) in a systematic epsilon expansion [10]:

$$
\begin{aligned}
I_{ \pm}= & -\frac{2 u_{0}^{2}}{9} \int \prod_{i=1}^{3} \frac{d^{d} \mathbf{k}_{i}}{(2 \pi)^{d} 2 \varepsilon_{k_{i}}}\left(\frac{\mathcal{F}_{ \pm}^{\text {out }}-\mathcal{F}_{ \pm}^{\text {in }}}{2 \varepsilon_{k}}\right)(2 \pi)^{d+1} \\
& \times \delta\left(\mathbf{k}+\mathbf{k}_{1}-\mathbf{k}_{2}-\mathbf{k}_{3}\right) \delta\left(\varepsilon+\varepsilon_{1}-\varepsilon_{2}-\varepsilon_{3}\right),
\end{aligned}
$$

where scattering out of state $\mathbf{k}$ is given by

$$
\begin{aligned}
\mathcal{F}_{ \pm}^{\text {out }}= & 2 f_{ \pm}(\mathbf{k}) f_{\mp}\left(\mathbf{k}_{1}\right)\left[1+f_{ \pm}\left(\mathbf{k}_{2}\right)\right]\left[1+f_{\mp}\left(\mathbf{k}_{3}\right)\right] \\
& +f_{ \pm}(\mathbf{k}) f_{ \pm}\left(\mathbf{k}_{1}\right)\left[1+f_{ \pm}\left(\mathbf{k}_{2}\right)\right]\left[1+f_{ \pm}\left(\mathbf{k}_{3}\right)\right],
\end{aligned}
$$

and we have suppressed the time dependence of the distributions. Scattering into state $\mathbf{k}$ follows by interchanging $f_{ \pm}$and $1+f_{ \pm}$. As usual, in order to access the critical region, the couplings must be tuned appropriately. Here

$$
m^{2}=\frac{4 \pi^{2} T^{2} \epsilon}{15}, \quad u_{0}=\frac{24 \pi^{2} \epsilon}{5},
$$

where $\epsilon=3-d$ and $T$ is the temperature [10]. The structure of the collision term (4) may also be seen using Fermi's golden rule, where the $1+f$ factors remind us that we are dealing with bosons. In this representation the electric current takes the form

$$
\mathbf{J}_{e}=Q \int \frac{d^{d} k}{(2 \pi \hbar)^{d}} \mathbf{v}_{\mathbf{k}}\left[f_{+}(\mathbf{k}, t)-f_{-}(\mathbf{k}, t)\right]
$$

and the heat current is given by

$$
\mathbf{J}_{h}=\int \frac{d^{d} k}{(2 \pi h)^{d}} \mathbf{v}_{\mathbf{k}} \varepsilon_{\mathbf{k}}\left[f_{+}(\mathbf{k}, t)+f_{-}(\mathbf{k}, t)\right]
$$

We have used the fact that within linear response, the heat current coincides with the energy current. The transport coefficients of interest are defined by

$$
\left(\begin{array}{c}
\mathbf{J}_{e}^{\mathrm{tr}} \\
\mathbf{J}_{h}^{\mathrm{tr}}
\end{array}\right)=\left(\begin{array}{ll}
\sigma & \alpha \\
\tilde{\alpha} & \bar{\kappa}
\end{array}\right)\left(\begin{array}{c}
\mathbf{E} \\
-\boldsymbol{\nabla} T
\end{array}\right) .
$$

Here, $\tilde{\alpha}=T \alpha$ is required by the Onsager relations. We denote the thermal transport coefficient, $\bar{\kappa}$, in order to distinguish it from the thermal conductivity defined in the absence of electrical transport. In order to work below $d=$ 3 in the $\epsilon$ expansion, we restrict ourselves to $\mathbf{E}$ and $\boldsymbol{\nabla} T$ orthogonal to $\mathbf{B}$, and to transport in the " $x y$ " plane defined by $\mathbf{E}$ and $\mathbf{E} \times \mathbf{B}$. Particle-hole symmetry places constraints on these remaining coefficients: the longitudinal component of $\alpha$ and the transverse (Hall) components of $\sigma$ and $\bar{\kappa}$ must vanish. Finally, we note that here, as in the standard Boltzmann treatment of magnetothermoelectric transport, we do not explicitly consider magnetization currents induced by the magnetic field $[5,13]$. We will discuss the implicit cancellations behind this elsewhere [14].

Separate response. - Before we turn our attention to the general problem in combined fields, let us first discuss what happens when each of the fields, $\mathbf{E}, \boldsymbol{\nabla} T$, and $\mathbf{B}$, is taken separately. The application of an electric field at finite temperatures leads to a linear response conductivity $\sigma_{x x}$ calculated in [10]. While the model thus supports current relaxation, it does not support energy current relaxation; it is known that its response to an applied temperature gradient - the thermal transport coefficient $\bar{\kappa}_{x x}$ is infinite [15]. The inclusion of the highly irrelevant umklapp scattering, reminiscent of the one-dimensional problems studied in [16], will ultimately render this quantity finite, though anomalously large as $T \rightarrow 0$. Finally, let us now turn to the response to a magnetic field taken alone. In the quantum critical region, and in the absence of $\mathbf{E}$ and $\boldsymbol{\nabla} T$, the only relevant energy scales are the temperature and the magnetic field. On general grounds we expect the free energy density to scale as

$$
\mathcal{F}(T, B)=T^{1+d / z} f_{1}\left(\frac{B}{T^{2 / z}}\right),
$$

where $f_{1}$ is some scaling function. Here we have used the fact that the correlation length $\xi$ diverges with the corre- 
lation time $\xi_{t} \sim 1 / T$ according to $\xi \sim\left(\xi_{t}\right)^{1 / z}$. The prefactor is thus an energy density. In addition we have used the fact that the vector potential $|\mathbf{A}| \sim 1 / L$, as may be seen from its gauge transformations, and so $|\mathbf{B}| \sim 1 / L^{2} \sim T^{2 / z}$. It follows that the linear response magnetization, for example, scales as

$$
M=-\frac{\partial \mathcal{F}}{\partial B} \sim T^{1+(d-4) / z} B .
$$

This is consistent with a finite temperature, diagrammatic Kubo calculation of the magnetic susceptibility of a charged scalar field, with $m \sim T$ and $z=1$ [14].

Crossed $\mathbf{E}$ and $\mathbf{B}$ fields. - The motion of a single relativistic charged particle in crossed electric and magnetic fields has two distinct regimes of behavior [17]. In the regime where $|\mathbf{E}|<c|\mathbf{B}|$ one may always find a frame moving with the velocity

$$
\mathbf{v}_{D}=\frac{\mathbf{E} \times \mathbf{B}}{|\mathbf{B}|^{2}}
$$

where the electric field vanishes. In this moving frame the particle executes cyclotron orbits. Boosting back to the lab frame the trajectories are helical, with a well-defined, charge independent, transverse drift velocity (11). In the regime $|\mathbf{E}|>c|\mathbf{B}|$ it is possible to find a frame where the magnetic field vanishes, but it is no longer possible to make the electric field vanish. In consequence, in the absence of any dissipative processes, the particle is continually accelerated by the electric field. In the lab frame the trajectories are hyperbolic. Since we are interested in linear response in a background magnetic field, we shall focus primarily on the drift regime; we comment on the hyperbolic regime later. The speed of light at issue here is the bosonic mode velocity suppressed in Eq. (2).

Drift regime $|\mathbf{E}|<c|\mathbf{B}|$. - Thus far we have used the Lorentz transformations to note some properties in the absence of the collision term. However, the Lorentz structure also enables us to make progress with the full QBE (3). As discussed above, the electric field vanishes in a frame moving with velocity $\mathbf{v}_{D}$. Since a magnetic field does not affect the energy of a particle it follows that a thermal distribution holds in this frame. Using the Lorentz transformation for energy, this suggests that

$$
f_{ \pm}(\mathbf{k})=f_{0}\left(\varepsilon_{k}^{\prime}\right)=f_{0}\left(\frac{\varepsilon_{k}-\mathbf{v}_{D} \cdot \mathbf{k}}{\sqrt{1-v_{D}^{2} / c^{2}}}\right),
$$

is a solution of the full QBE (3), including the collision term. This remarkable fact may be verified by explicit substitution. In particular, we may Taylor expand (12) to linear order in $\mathbf{v}_{D}$, or equivalently the electric field. Substituting this into (7) yields a nonvanishing transverse heat current, and a thermoelectric tensor

$$
\alpha_{x y}=\frac{2 c^{2}}{d B T} \int \frac{d^{d} k}{(2 \pi \hbar)^{d}} k^{2}\left(-\frac{\partial f_{0}}{\partial \varepsilon_{k}}\right) .
$$

This result also follows from entropy drift [14]. In writing (13) we have employed the Onsager relation $\tilde{\alpha}=T \alpha$; we justify this below. Note that we may also make contact with (12) from a direct linearization of the QBE. Substituting the decomposition

$$
f_{ \pm}(\mathbf{k})=f_{0}\left(\varepsilon_{k}\right) \pm Q \mathbf{k} \cdot \mathbf{E} \psi(k)+\mathbf{k} \cdot(\mathbf{E} \times \mathbf{B}) \psi_{\perp}(k)
$$

into Eq. (3) and linearizing in the electric field, yields $\psi(k)=0$, and an expression for $\psi_{\perp}(k)$ consistent with the Taylor expansion of (12). Having established the validity of (13) as a legitimate result for the full QBE, to lowest order in the epsilon expansion we compute the numerical prefactor in $d=3$ and for $m=0$ :

$$
\alpha_{x y}=\frac{4 \pi^{2}}{45} \frac{k_{B}}{B}\left(\frac{k_{B} T}{\hbar c}\right)^{3-\epsilon} \text {. }
$$

This is consistent with scaling near a quantum critical point [14]

$$
\alpha_{x y}(T, B) \sim T^{(d-2) / z} f_{2}\left(\frac{B}{T^{2 / z}}\right) \sim \frac{T^{d}}{B},
$$

where $f_{2}$ is another scaling function. Note that the dependence on $1 / B$, which stems from the drift velocity (11), is analogous to that of the clean, single carrier classical Hall conductivity. It is important to recall, however, that $\sigma_{x y}=$ 0 , in our particle-hole symmetric case. Likewise, $\sigma_{x x}=0$ in this regime, owing to the purely transverse nature of (12). Having completed our analysis in the drift regime, let us discuss the transport properties in the presence of a thermal gradient and a magnetic field.

Crossed $\boldsymbol{\nabla}$ T and $\mathbf{B}$ fields. - In keeping with our previous discussion, let us consider $|\nabla T| \ll|\mathbf{B}|$. We may introduce a temperature gradient into the QBE by allowing the temperature variable to be a function of position [18]. The generic $\mathrm{QBE}$, with $\mathbf{E}=0$, reads

$$
\frac{\partial f_{ \pm}}{\partial t}+\mathbf{v}_{k} \cdot \frac{\partial f_{ \pm}}{\partial \mathbf{x}} \pm Q\left(\mathbf{v}_{\mathbf{k}} \times \mathbf{B}\right) \cdot \frac{\partial f_{ \pm}}{\partial \mathbf{k}}=I_{ \pm}\left[f_{+}, f_{-}\right] .
$$

In the absence of material inhomogeneity, we assume that any spatial variation is due to $\nabla T$ :

$$
\frac{\partial f_{ \pm}}{\partial \mathbf{x}}=\nabla T\left(\frac{\partial f_{ \pm}}{\partial T}\right)=\nabla T\left(-\frac{\varepsilon_{k}}{T} \frac{\partial f_{0}}{\partial \varepsilon_{k}}\right) .
$$

In the last step we have used the fact that we are interested in linear response in $\nabla T$. To solve the resulting linearized equation we parametrize

$$
f_{ \pm}(\mathbf{k})=f_{0}\left(\varepsilon_{k}\right)+\mathbf{k} \cdot \mathbf{U} \psi(k) \pm Q \mathbf{k} \cdot(\mathbf{U} \times \mathbf{B}) \psi_{\perp}(k),
$$

where $\mathbf{U} \equiv-\boldsymbol{\nabla} T / T$. To lowest order in $\epsilon$, we may drop the collision term:

$$
\psi(k)=0, \quad \psi_{\perp}(k)=\frac{\varepsilon_{k}}{Q^{2}|\mathbf{B}|^{2}}\left(-\frac{\partial f_{0}}{\partial \varepsilon_{k}}\right) .
$$

This reproduces our previous result (13) as expected from Onsager reciprocity. Proceeding to $\mathcal{O}\left(\epsilon^{2}\right)$ we find a nonvanishing longitudinal displacement

$$
\psi(k)=\epsilon^{2}\left(\frac{\varepsilon_{k}}{\hbar c}\right) \int_{0}^{\infty} d k_{1}\left[\psi_{\perp}(k) F_{1}+\psi_{\perp}\left(k_{1}\right) F_{2}\right]
$$


where $\psi_{\perp}(k)$ is given by our zeroth order result (20). The kernels $F_{1}\left(k, k_{1}\right)$ and $F_{2}\left(k, k_{1}\right)$ follow immediately upon direct substitution of (19) into the collision term (4). Linearizing in $\nabla T$, they emerge quite naturally as integrals over products of the remaining Bose distribution functions. These may be evaluated using polylogarithms [10], but their detailed form need not concern us here. Introducing the dimensionless variable $\bar{k}=c k / k_{B} T$, we may recast this longitudinal displacement as

$$
\psi(k)=\frac{\epsilon^{2}}{\hbar}\left(\frac{k_{B} T}{Q B C}\right)^{2} G(\bar{k}),
$$

where

$$
G(\bar{k}) \equiv \bar{k} \int_{0}^{\infty} d \bar{k}_{1}\left[P(\bar{k}) \Phi_{1}\left(\bar{k}, \bar{k}_{1}\right)+\Phi_{2}\left(\bar{k}, \bar{k}_{1}\right) P\left(\bar{k}_{1}\right)\right] .
$$

Here, $P(\bar{k})=\bar{k} e^{\bar{k}} /\left(e^{\bar{k}}-1\right)^{2}$ is the massless limit of $\psi_{\perp}(k)$, and $\Phi_{i}\left(\bar{k}_{1}, \bar{k}_{2}\right) \equiv F_{i}\left(k_{1}, k_{2}\right)$ are rescaled kernels. Upon integration over momentum space, the longitudinal displacement (22) leads to a finite thermal transport coefficient

$$
\bar{\kappa}=g \epsilon^{2}\left(k_{B} c\right) l_{B}^{4} \lambda_{T}^{-(d+3)},
$$

where $l_{B} \equiv(\hbar / Q B)^{1 / 2}$ and $\lambda_{T} \equiv \hbar c / k_{B} T$ are the magnetic length and a suitable thermal wavelength, respectively. To lowest order in the epsilon expansion

$$
g=\frac{1}{3 \pi^{2}} \int_{0}^{\infty} d \bar{k} \bar{k}^{4} G(\bar{k}) \approx 5.55 .
$$

This is in contrast to the zero magnetic field case, where the response of the clean system is infinite.

Beyond the linear Boltzmann regime. - Above we have considered linear transport response in $\mathbf{E}$ and $\boldsymbol{\nabla} T$ with a finite $B \ll T$ already present. This is what is naturally described by the linearized QBE. Evidently, this is not all the behavior that is possible in the quantum critical regime: with $T \gg E, B, \nabla T$, it is still possible to have other ratios for the three applied fields. We have previously noted that for $E>c B$ single particle orbits are fundamentally different. One can show more generally that the drift solution fails to exist in this domain, even in the presence of the interactions in the fixed point theory (2). It seems clear that the fixed point theory should yield a finite $\sigma_{x x}$, and an infinite $\alpha_{x y}$ in this regime, but a formal demonstration needs to be constructed. The situation with an applied $\boldsymbol{\nabla} T$ is similar, but in need of more work. Specifically, the boundary between the $\nabla T \ll B$ regime with a finite $\bar{\kappa}_{x x}$, and the $\nabla T \gg B$ regime which is expected to exhibit an infinite $\bar{\kappa}_{x x}$, although finite $\alpha_{x y}$, cannot be located with precision by our methods. We hope to report progress on these issues elsewhere [14].

Concluding remarks. - We have examined the magnetothermoelectric response at the SF-MI transitions of the Bose-Hubbard model. In linear transport in an applied magnetic field, we obtain a finite thermoelectric tensor and thermal transport coefficient. It is worth noting that in contrast to the $B=0$ electrical conductivity [10] these coefficients are regular in the epsilon expansion. Finally, we comment on a Nernst measurement in such a system in which we measure a transverse electric field induced by a thermal gradient in an open electrical circuit. For the measurement to be well defined, we will need to invoke irrelevant umklapp processes to regularize quantities that are infinite in the fixed point theory. This done, we find that in the scaling limit an infinitesimal $\nabla T$ induces a finite Nernst electric field of strength $B / c$ [14].

We are extremely grateful to D. Basko, J.-S. Caux, J. Chalker, K. Damle, F. Essler, C. Hooley, A. Lamacraft, S. Sachdev, V. Oganesyan, and A. Tsvelik for valuable input at various stages. We are especially indebted to David Huse for insightful comments on the Lorentz transformations for $E>c B$, and S. Sachdev for additional helpful suggestions while this manuscript was in proof. This work was supported by NSF Grant No. DMR0213706, EPSRC, and The Royal Society. M. J. B. would also like to thank BNL and St. Andrews for hospitality during part of this work.

[1] M. Greiner, O. Mandel, T. Esslinger, T. W. Hänsch, and I. Bloch, Nature (London) 415, 39 (2002).

[2] S. Sachdev, Quantum Phase Transitions (Cambridge University, Cambridge, 1999).

[3] Z. A. Xu, N. P. Ong, Y. Wang, T. Kakeshita, and S. Uchida, Nature (London) 406, 486 (2000).

[4] Y. Wang, L. Li, M. J. Naughton, G. D. Gu, S. Uchida, and N. P. Ong, Phys. Rev. Lett. 95, 247002 (2005).

[5] I. Ussishkin, S. L. Sondhi, and D. A. Huse, Phys. Rev. Lett. 89, 287001 (2002).

[6] A. S. Sørensen, E. Demler, and M. D. Lukin, Phys. Rev. Lett. 94, 086803 (2005).

[7] M. P. A. Fisher, P. B. Weichman, G. Grinstein, and D. S. Fisher, Phys. Rev. B 40, 546 (1989).

[8] M. P. A. Fisher, G. Grinstein, and S. M. Girvin, Phys. Rev. Lett. 64, 587 (1990).

[9] M.-C. Cha, M. P. A. Fisher, S. M. Girvin, M. Wallin, and A. P. Young, Phys. Rev. B 44, 6883 (1991).

[10] K. Damle and S. Sachdev, Phys. Rev. B 56, 8714 (1997).

[11] A. G. Green and S. L. Sondhi, Phys. Rev. Lett. 95, 267001 (2005).

[12] A. G. Green, J.E. Moore, S. L. Sondhi, and A. Vishwanath, cond-mat/0605615.

[13] N. R. Cooper, B. I. Halperin, and I. M. Ruzin, Phys. Rev. B 55, 2344 (1997).

[14] M. J. Bhaseen, A. G. Green, and S. L. Sondhi (to be published).

[15] M. Vojta, Y. Zhang, and S. Sachdev, Int. J. Mod. Phys. B 14, 3719 (2000); T. Senthil (unpublished).

[16] E. Shimshoni, N. Andrei, and A. Rosch, Phys. Rev. B 68, 104401 (2003).

[17] J.D. Jackson, Classical Electrodynamics (Wiley, New York, 1998), 3rd ed.

[18] J.M. Ziman, Principles of the Theory of Solids (Cambridge University, Cambridge, 1964). 